\title{
IR-UWB Radar Sensor for Fine Human Motion Detection
}

\author{
Razak Mohd Ali Lee ${ }^{1 *}$, Terence Jerome Daim² \\ ${ }^{1}$ Faculty of Engineering, \\ Universiti Malaysia Sabah, Kota Kinabalu, 88400, MALAYSIA \\ ${ }^{2}$ Faculty of Engineering, \\ Universiti Malaysia Sabah, Kota Kinabalu, 88400, MALAYSIA \\ *Corresponding Author
}

DOI: https://doi.org/10.30880/ijie.2020.12.07.001

Received 9 March 2020; Accepted 3 August 2020; Available online 30 August 2020

\begin{abstract}
Fine human motion detection in contact-less manner based on radar technology is increasingly appealing research efforts among researchers and one of the promising approach that have garnered research momentum in recent years is IR-UWB radar. IR-UWB radar is a kind of radar sensor that was developed from combining UWB and radar technology. Being a technology that offers unique capability of penetrability, high temporal resolution, low power consumption and emits non-ionizing electromagnetic waves, IR-UWB radar sensor is an encouraging fusion of radar and UWB technology that can be leveraged into many useful extensive number of application. In this paper, a preliminary study of detecting fine human motion using IR-UWB radar sensor is presented where a system model based on the IR-UWB radar sensor operation on fine human motion detection is described. For testing, this work employs a right hand and fingers. Based on the tests, the movement of right hand and fingers can be discriminated accurately at a distance of between $5 \mathrm{~cm}$ to $10 \mathrm{~cm}$ with minor occurrence of noises.
\end{abstract}

Keywords: IR-UWB, fine human motion detection, radar

\section{Introduction}

In general, a radar system function based on the concept of Radio Frequency (RF) electromagnetic waves detection and reflection [1]. The array of RF within the electromagnetic spectrum has numerous greatly striking features that enable viable and robust sensing capability for various applications and interactive systems. One feature would be the capability to function without ideal illumination or atmospheric environment, which is useful in dusty and not well lit area of operation. Other features would be that they are able to function at a very fast and highly accurate manner and can operate across materials that enable them to be conveniently incorporated as on-chip devices and environments.

The whole system can be engineered as a lightweight solid-state semiconductor package when applied at millimeter-wave RF frequencies. Thus resulting a radar processor that is small, low-power and no moving components device which can be produced at low-cost. RF sensing has become a well-established and developed field of engineering and applied science since the earliest ever radar systems were developed in the early 1930s by [2]. The latest state-of-the-art radar equipment and computing techniques were developed predominantly for conventional radar applications, typically involving identification and analysis of big moving targets at great ranges such as air and ground traffic control etc. The engineering involves for these conventional radar application are not well-suited with modern consumer applications whereby sensor must meet several criteria such as size, portability, low power consumption and limited processing resources to sense complex, fine human motion in contact-less manner as opposed to rigid object. Such sensing capability based on radar technology is increasingly appealing research efforts among researchers and one 
of the promising approach that have garnered research momentum in recent years is IR-UWB (Impulse Radio-Ultra Wide Band) radar.

IR-UWB radar is a type of radar sensor which was developed from combining UWB and radar technology. By utilizing a wideband signal configuration coupled with a small Equivalent Isotopically Radiated Energy (EIRP) of 41.3 $\mathrm{dBm} / \mathrm{MHz}$ in the frequency range of 3.1 to $10.6 \mathrm{GHz}$ (ET Docket 98-153, First Report and Order 02-48), the United States of America's Federal Communications Commission (FCC) has sanctioned the unlicensed used of UWB technology in the year 2002. As defined by the FCC, a wireless signal is characteristically determined to be UWB if its absolute bandwidth exceeds $500 \mathrm{MHz}$. Being a technology that offers unique capability of penetrability, high temporal resolution [3]-[8], low power consumption and emits non-ionizing electromagnetic waves [9], IR-UWB radar sensor is an encouraging fusion of radar and UWB technology that can be leveraged into many useful extensive number of application. These applications are such as rescue operation in post-disaster scenario, contactless measurement for medical purposes and through-wall surveillance [10]-[14]. IR-UWB radar sensor are also very immune from other kinds of wireless radio system in its 3.1 to $10.6 \mathrm{GHz}$ band while causes only negligible interference to others [15]-[18].

In this paper, a preliminary study to detect fine human motion using IR-UWB radar sensor is described. This paper is structured as follows. In Section 2, the fundamental operation of an IR-UWB radar sensor is presented while Section 3 described the system model for fine human motion detection using IR-UWB radar sensor. In Section 4, the test setup is presented while Section 5 provides the results and discussion. The conclusion and future work are lastly presented in Section 6.

\section{Fundamental Operation of An IR-UWB Radar Sensor}

Referring to Fig. 1, the fundamental operation of an IR-UWB radar sensor is depicted. The IR-UWB radar sensor first transmit out electromagnetic impulses or in this case UWB impulses through the IR-UWB radar sensor's transmitting (Tx) antenna which is reflected from any object in front of it. The reflected UWB impulses then travels back and are received through the IR-UWB radar sensor's receiving (Rx) antenna. When the IR-UWB radar sensor received the reflected UWB impulses, it will be sampled by going through an analog to digital converter before being fed into a bandpass filter.

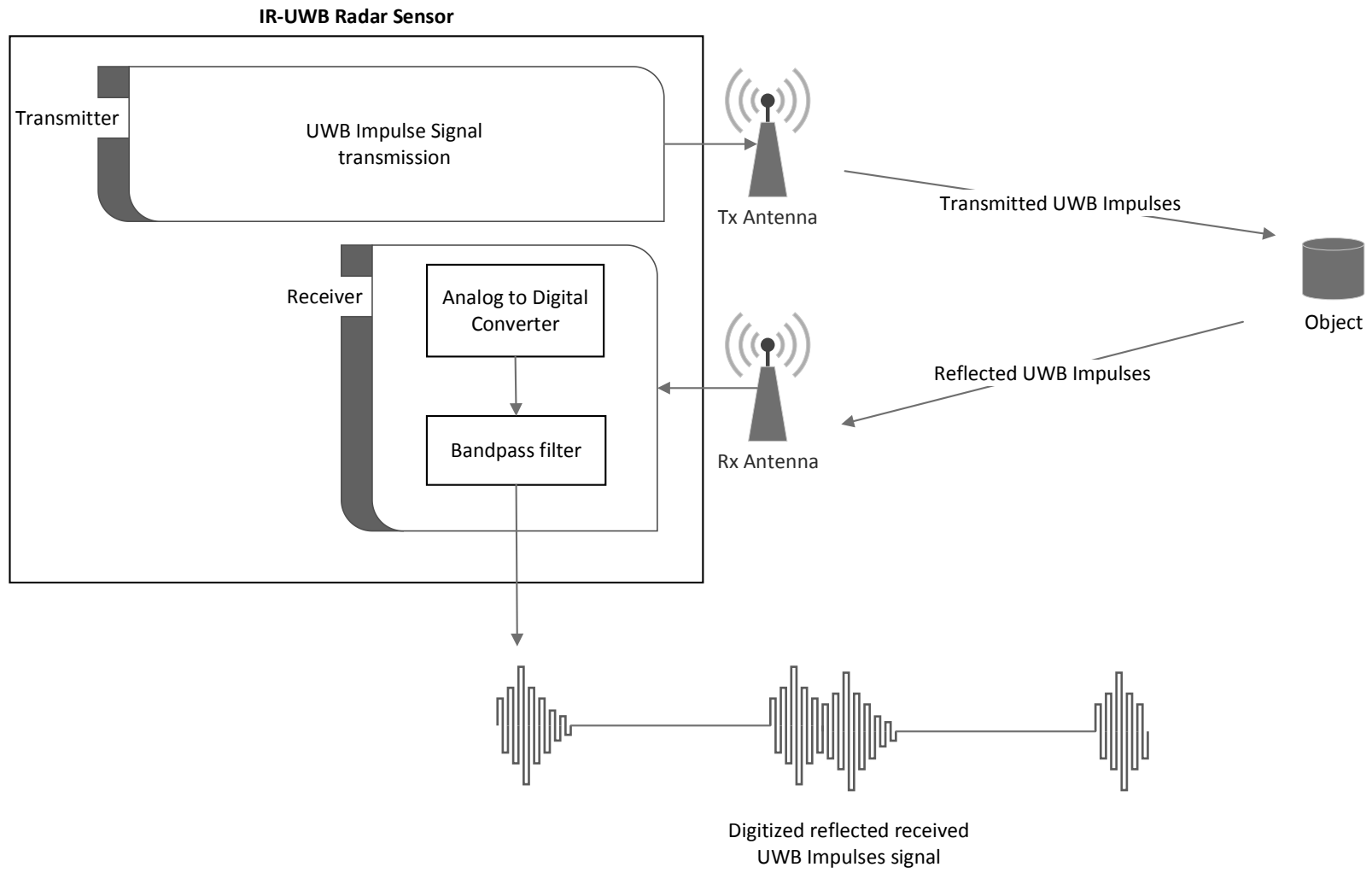

Fig. 1 - Fundamental operation of IR-UWB radar sensor

In terms of sampling capability, the IR-UWB radar sensor used in this work is capable of handling a sampling rate of up to $23.328 \mathrm{G}$ sample/s that can sample up to a maximum of 1536 samples. The sampling of reflected UWB impulses corresponds to a range of approximately 9.9 meters long. This is the case since the speed of electromagnetic 
waves travels is equivalent to the speed of light. Referring to Fig. 2, a range bin is being referred for each sampling point within the range of the reflected UWB impulses and a collection of these range bins is called a radar frame (Fig. 3).

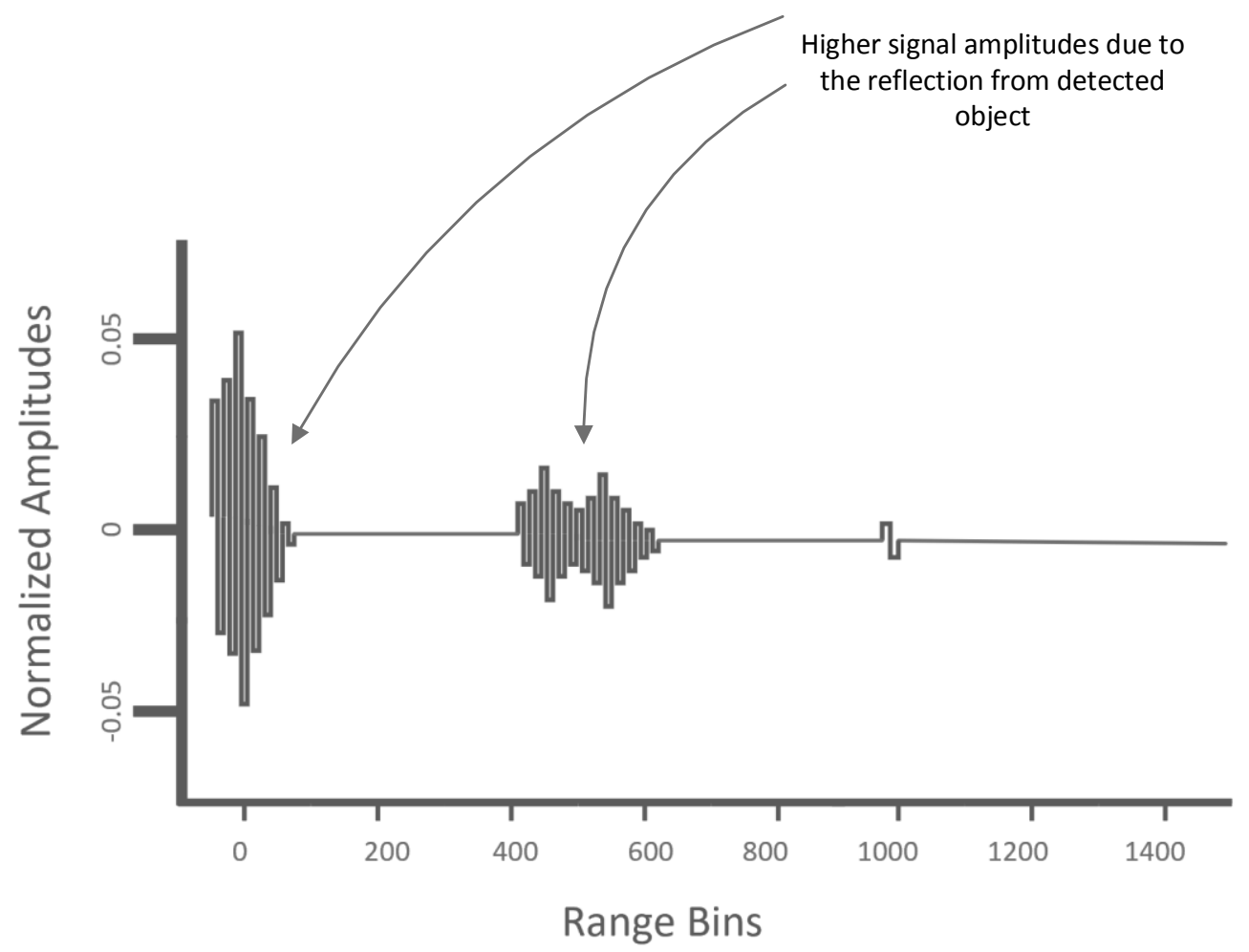

Fig. 2 - A radar frame

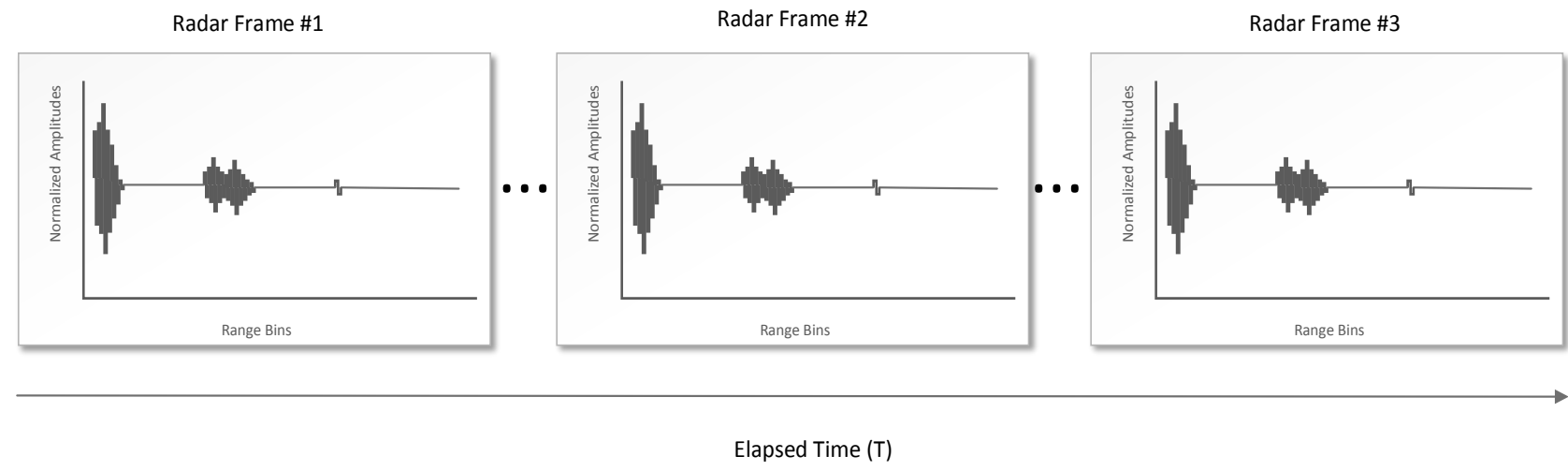

Fig. 3 - Recorded radar frame vs elapsed time (T)

When an object is detected, the distance from the IR-UWB radar sensor to the object is referred to as slant range and is calculated by equation (1) below:

$$
\text { Slant Range }=\frac{c \times \tau}{2}
$$

Where $\mathrm{c}$ is speed of light, $3 \times 10^{8} \mathrm{~m} / \mathrm{s}, \tau$ is the measured delay due to the reflected UWB pulses on the object and since the signal has to travels twice the distance to the target and back, a divisor of 2 has to be applied. 


\section{System Model}

The basic principle of fine human motion detection using IR-UWB radar sensor is based on the identification of reflected UWB impulses from the human body. The changes in signal amplitude as well as the Time of Arrival (ToA) of the reflected UWB impulse are used to detect and identify the various states of fine human motion. Referring to figure 4, when an UWB impulse is generated and transmitted, the reflected UWB impulse resulted from fine human motion is received by the IR-UWB radar sensor.

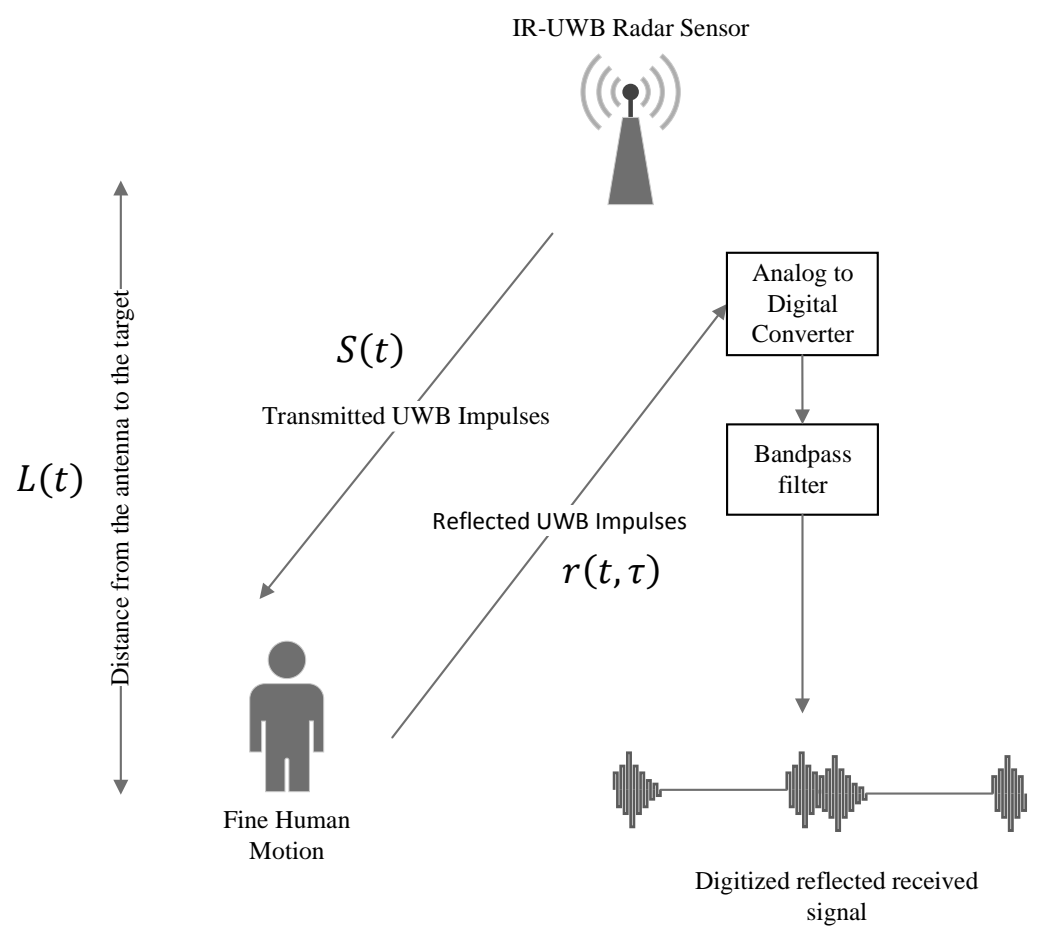

Fig. 4 - Fine Human Motion Detection Using IR-UWB Radar

The received UWB impulse signal is then being sampled by going through an analog to digital converter before being fed through a bandpass filter to produce a less noise digitized version of the signal. In this case, the range from the antenna to the target $L(t)$ can be described as equation (2):

$$
\begin{aligned}
L(t) & =d_{0}+d(t) \\
& =d_{0}+d_{i} \sin \left(2 \pi f_{i} t\right)
\end{aligned}
$$

Where $d_{0}$ the nominal distance between antenna and target is, $d_{i}$ is the displacement amplitude of target fine human motion and $f_{i}$ represents the fine motion frequency. The normalized received UWB impulse signal is represented by $\delta(t)$, and the total impulse response can be denoted by equation (3):

$$
r(t, \tau)=\sum_{m=1}^{N} a_{m} \delta\left(\tau-\tau_{m}(t)\right)
$$

Where $t$ denotes the observation interval and $\tau$ is the propagation interval. $\sum_{m=1}^{N} a_{m} \delta\left(\tau-\tau_{m}(t)\right)$ denotes the response due to fine human motion along with propagation interval $\tau_{m}(t)$ and amplitude $a_{m} \cdot \tau_{m}(t)$, is determined by the range from the antenna to the target, $L(t)$ and can be described as equation (4):

$$
\begin{aligned}
\tau_{m}(t) & =\frac{2 L(t)}{c} \\
& =\tau_{0}+\tau_{i} \sin \left(2 \pi f_{i} t\right)
\end{aligned}
$$

Where $c$ is the speed of light $=3 \times 10^{8} \mathrm{~m} / \mathrm{s}, \tau_{0}=2 d_{0} / c$ and $\tau_{i}=2 d_{i} / c$ 


\section{Test Setup}

In this work, two types of tests are conducted. The first test involves the investigation of distance vs target object position. The purpose of this test is to determine the distance in which an object can be detected while in motion with the least errors produced. The second test involves fine human motion detection based on the system model as described in section 3. The same IR-UWB radar sensor is used for both tests. The IR-UWB radar sensor is connected to a processing terminal for data processing. The IR-UWB radar sensor used in this work is shown in Fig. 5. The IR-UWB radar sensor is made by NOVELDA model X4.

(a)

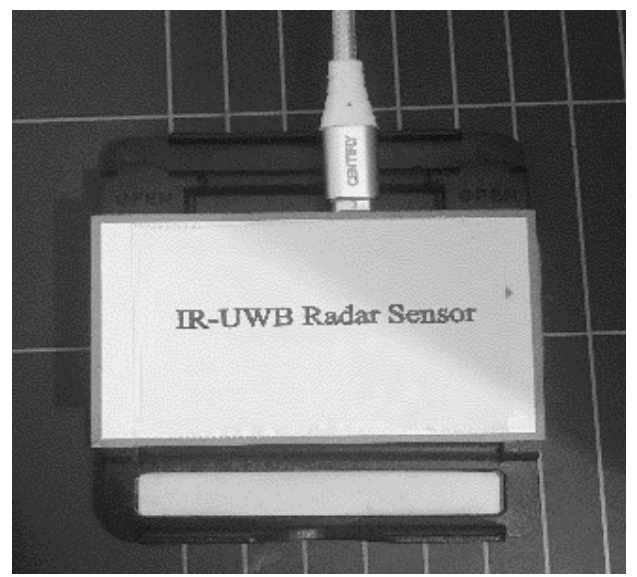

(b)

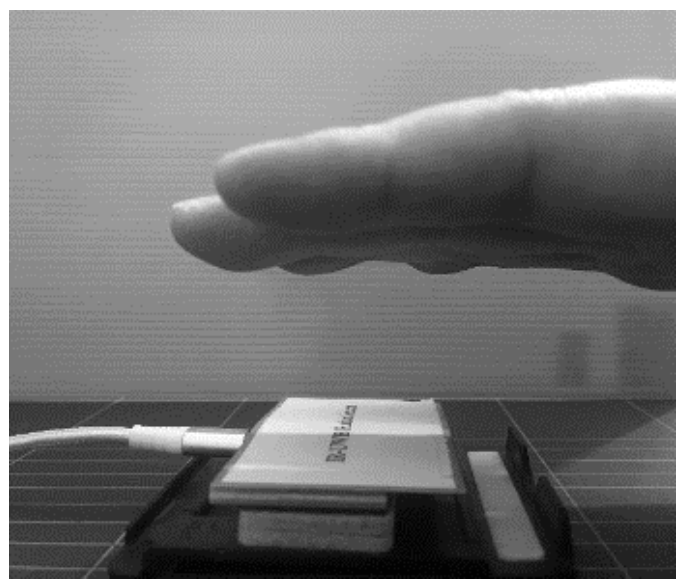

Fig. 5 - (a) IR-UWB radar sensor used in this work; (b) side view of the radar sensor

\subsection{Distance Between Detected Object and Radar Test}

In order to determine the distance between an object and the IR-UWB radar, Fig. 6 shows the test setup that was used. A right hand was employed as a target for the IR-UWB radar sensor to sense its present and measure the distance from the IR-UWB radar. For the purpose of this test, the measurement will start from $10 \mathrm{~cm}$ distance from the IR-UWB radar sensor and gradually increase with $10 \mathrm{~cm}$ interval until reaching maximum distance of $200 \mathrm{~cm}$. This work choose $200 \mathrm{~cm}$ as the maximum distance for this test as this is sufficient enough to cover most intended application. A software was developed to measure the delay due to the reflected UWB pulses on the right hand target. The distance or slant range are calculated by using equation (1) and can be extracted and processed from the IR-UWB radar sensor raw data. Fig.7 shows an example where the right hand is positioned in this test.

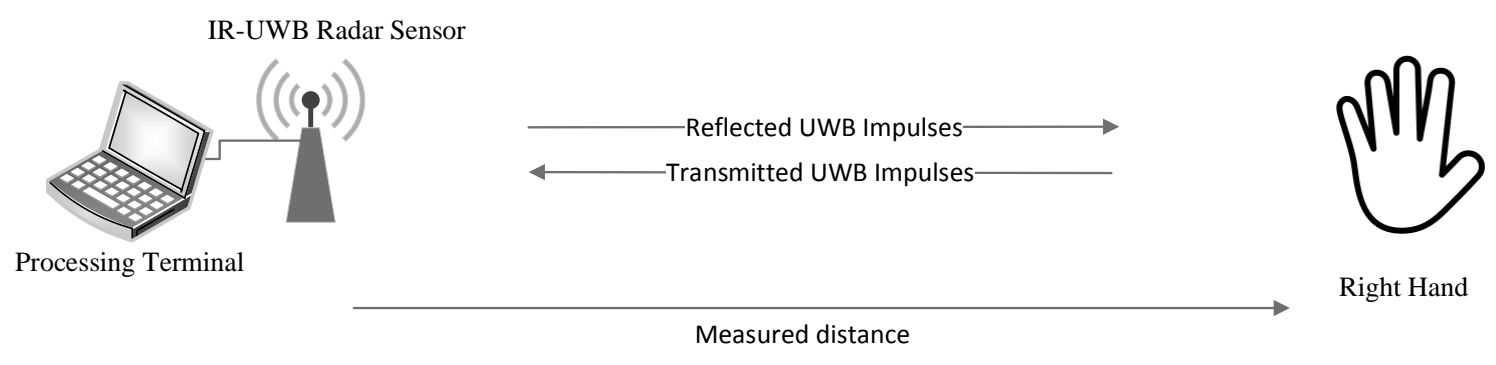

Fig. 6 - Test setup for distance between detected object and radar. 

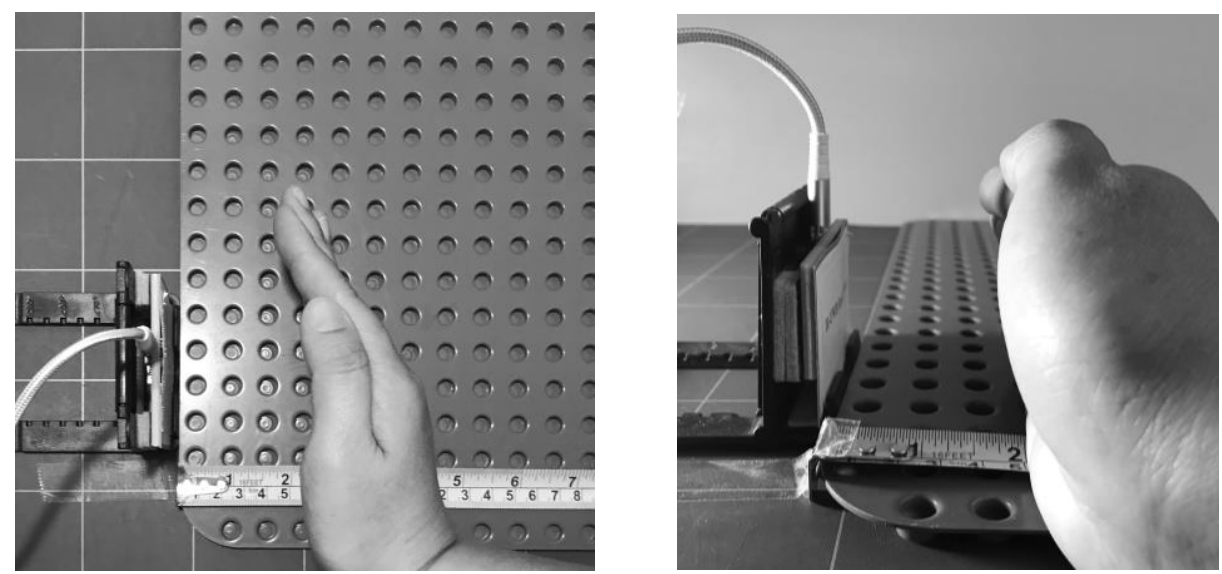

Fig. 7 - Example placement of right hand (a) top view; (b) side view

\subsection{Fine Human Motion Detection Test}

Fig. 8 depicts the test setup that is used in this fine human motion detection test, which is also the second test in this work. To detect fine human motion, this work has determine to detect and identify the fine movement of a right hand and fingers and can be further divided into two sub-tests. The first sub-test is to evaluate the system model along with the hardware implementation capability to react and discriminate accurately in accordance to the right hand placement off and on hovering on top of the IR-UWB radar sensor at exactly 100, 200 and 100\&200 IR-UWB radar frames interval (Fig. 8). The second sub-test is similar to the first sub-test however instead with right hand placement off and on hovering on top of the IR-UWB radar sensor, the right hand will stay put hovering all the time during the duration of the test and only varies with wiggling of fingers movement at 100, 200 and 100\&200 radar frames intervals (Fig. 9). The distance in which the right hand hovers on top of the IR-UWB radar sensor will be determine by the results conducted in the first test.

(a)

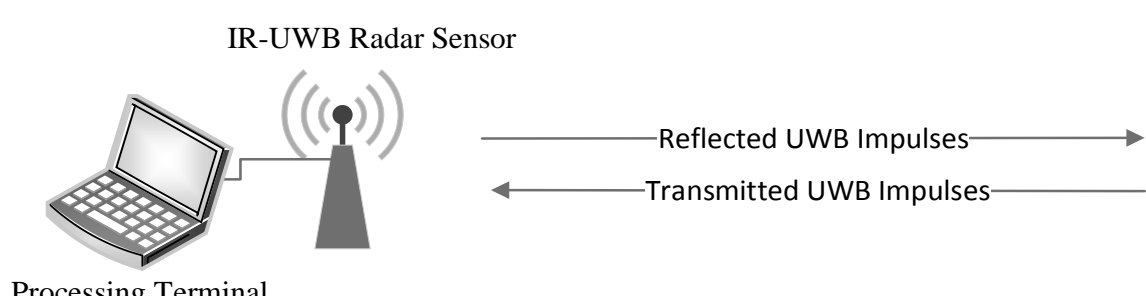

Processing Terminal

(b)

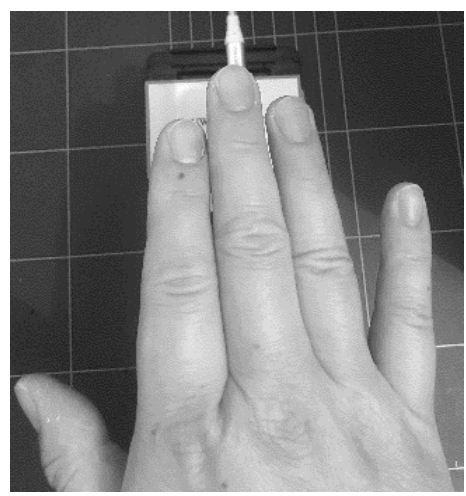

(c)

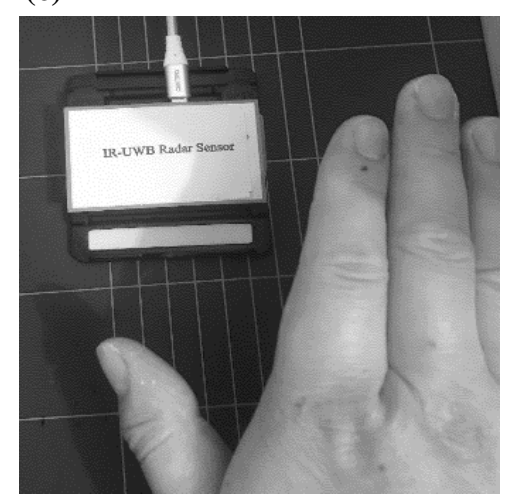

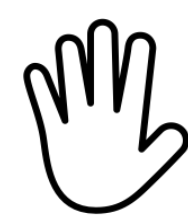

Right hand/Fingers Movement

Fig. 8 - (a) Test Setup, First sub-test (b) on-hand, (c) off-hand 


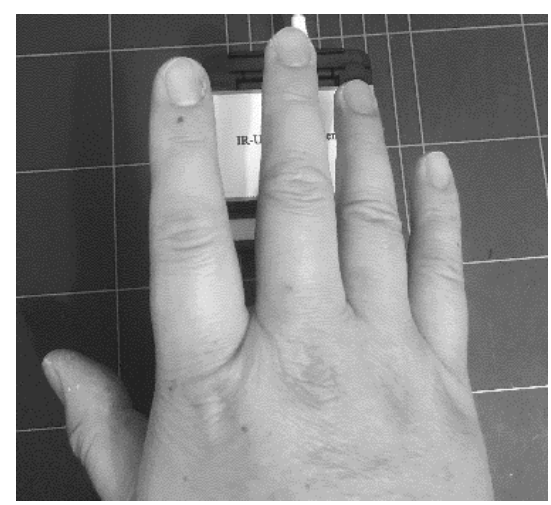

Fig. 9 - Second sub-test - wiggling of fingers movement

\section{Result and Discussion}

The test result for the first test is depicted in Fig. 10, the measurement of distance which are made by extracting and processing from the IR-UWB radar sensor raw data shows similar trend when compare to the distance measured using a measurement tape. However, significant errors occurs at several point of measurements. Notably at $35 \mathrm{~cm}, 45$ $\mathrm{cm}$ and from $105 \mathrm{~cm}$ onwards. The least errors recorded are from $5 \mathrm{~cm}$ till $25 \mathrm{~cm}$ and from $55 \mathrm{~cm}$ till $95 \mathrm{~cm}$. Therefore, based on these test results it can be determined that the distance in which an object can be detected while in motion with the least errors produced are within $5 \mathrm{~cm}$ till $25 \mathrm{~cm}$ and from $55 \mathrm{~cm}$ till $95 \mathrm{~cm}$. For the purpose of conducting the second test in this work, the distance in which the right hand hovers on top of the IR-UWB radar sensor will be between $5 \mathrm{~cm}$ to $10 \mathrm{~cm}$.

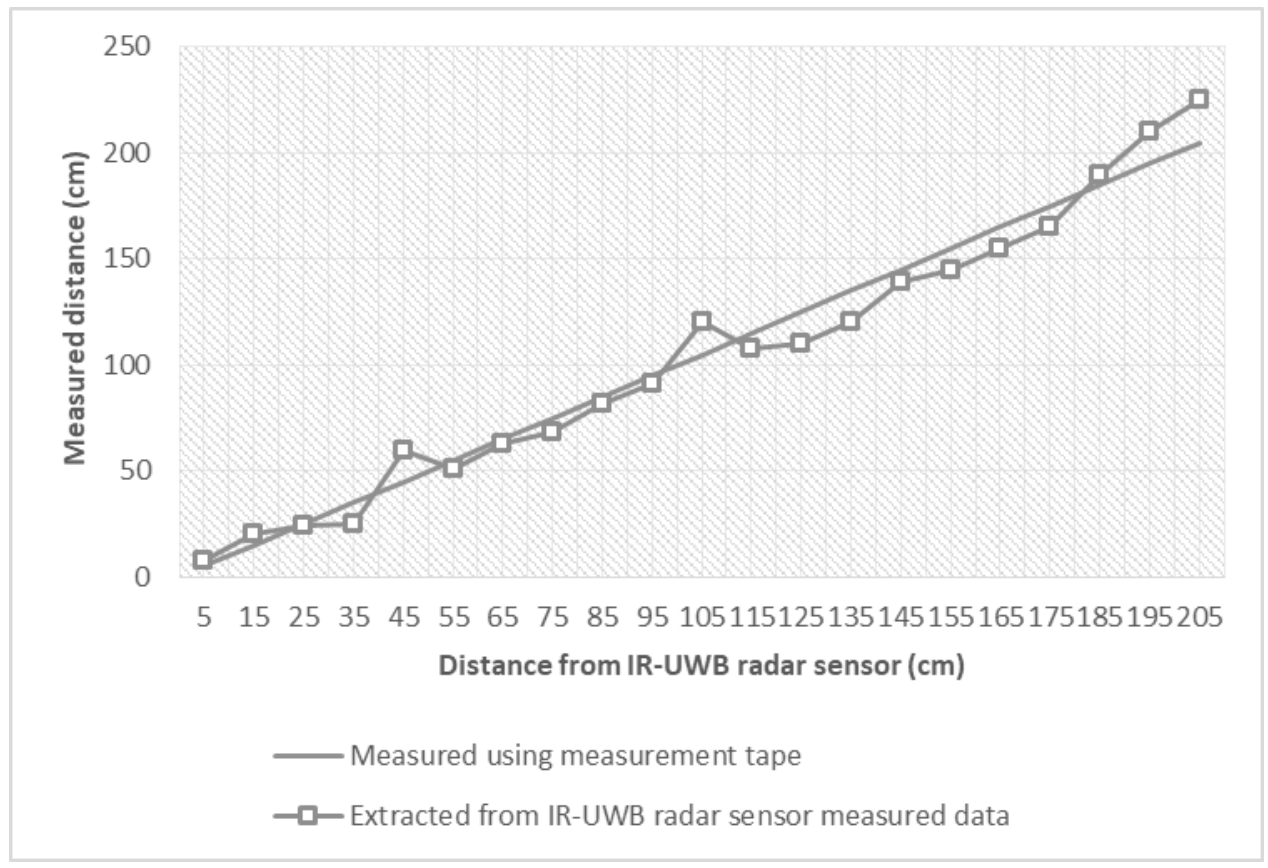

Fig. 10 - Measured distance using measurement tape vs distance extracted from IR-UWB radar sensor.

Fig. 11 depicts the second test's first sub-test result. As can be observed the clear separation of amplitudes change between on and off hand movement at 100, 200 and $100 \& 200$ radar frames respectively. This indicates the implemented system model is able to react and discriminate accurately according to the hand movement as found in Fig.8. In this case, higher amplitudes are recorded when the hand is hovering on top of the IR-UWB radar sensor and lower amplitudes when it is off the top of the IR-UWB radar sensor. The amplitude changes from high to low and low to high almost instantaneously when a hand movement occur for each of the intervals specified in this test. The same observation can be found on the second sub-test result as depicted in Fig. 12. However, the separations are not that 
obvious when compare to the first sub-test result. The results are rather noisy and at some points the amplitudes of several radar frames behaves radically. Overall based on these tests, it can be seen that the system model coupled with the implemented hardware responds well to fine human motion. Although generally that's the case, several enhancements have to be in cooperated in future works such as refinement of the algorithm in the system model which may include several noise suppression strategies.

(a)

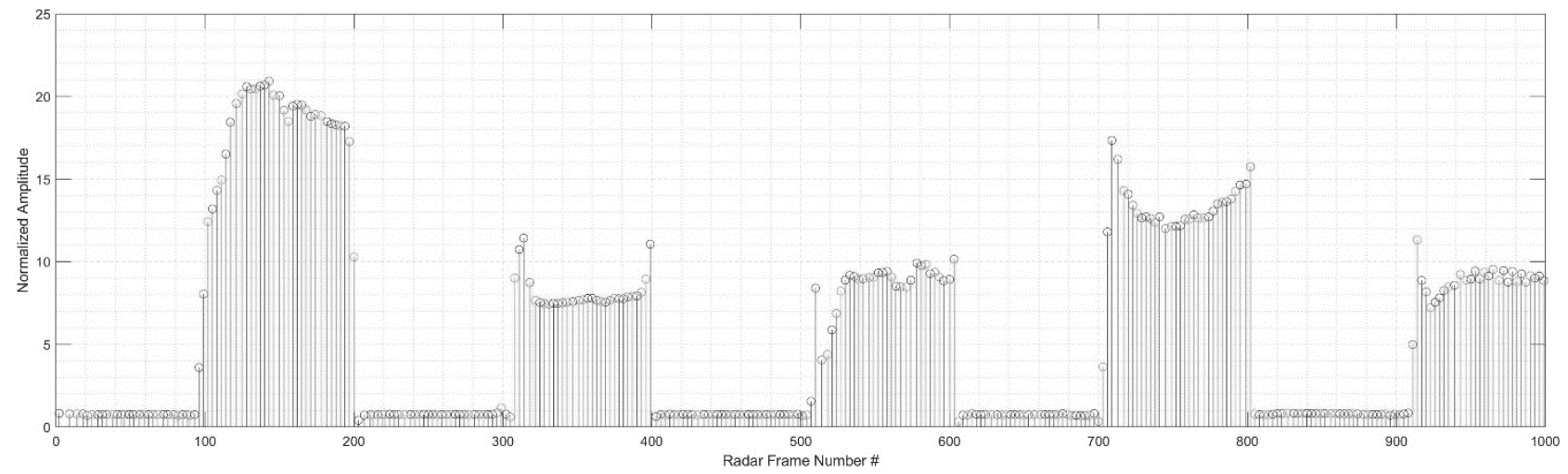

(b)

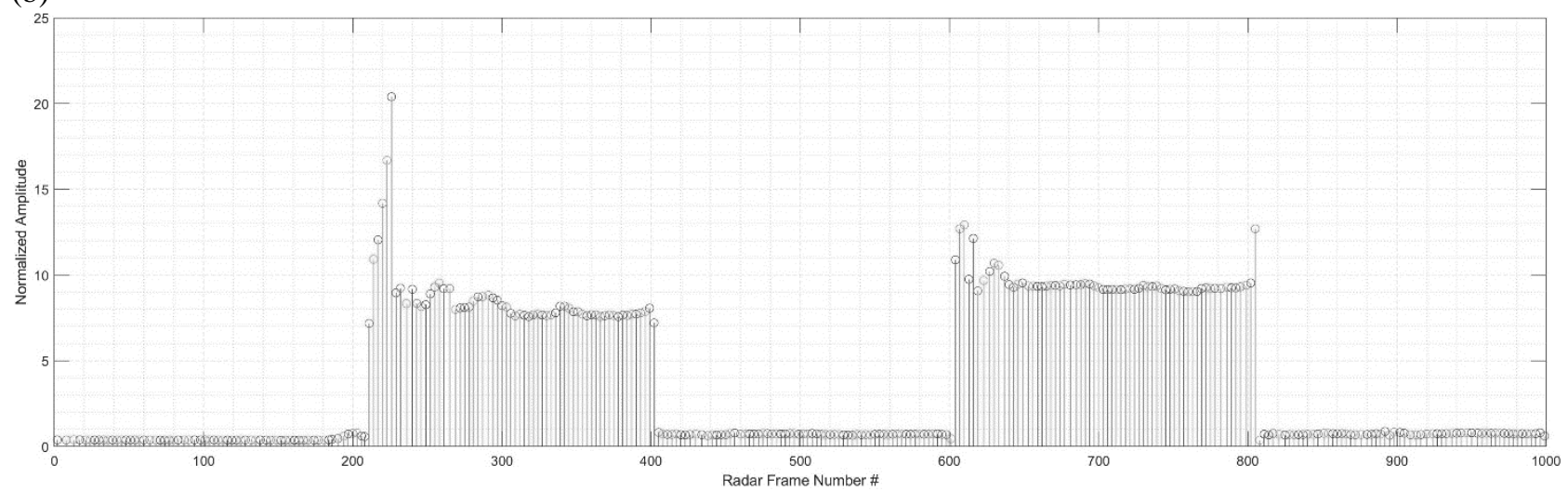

(c)

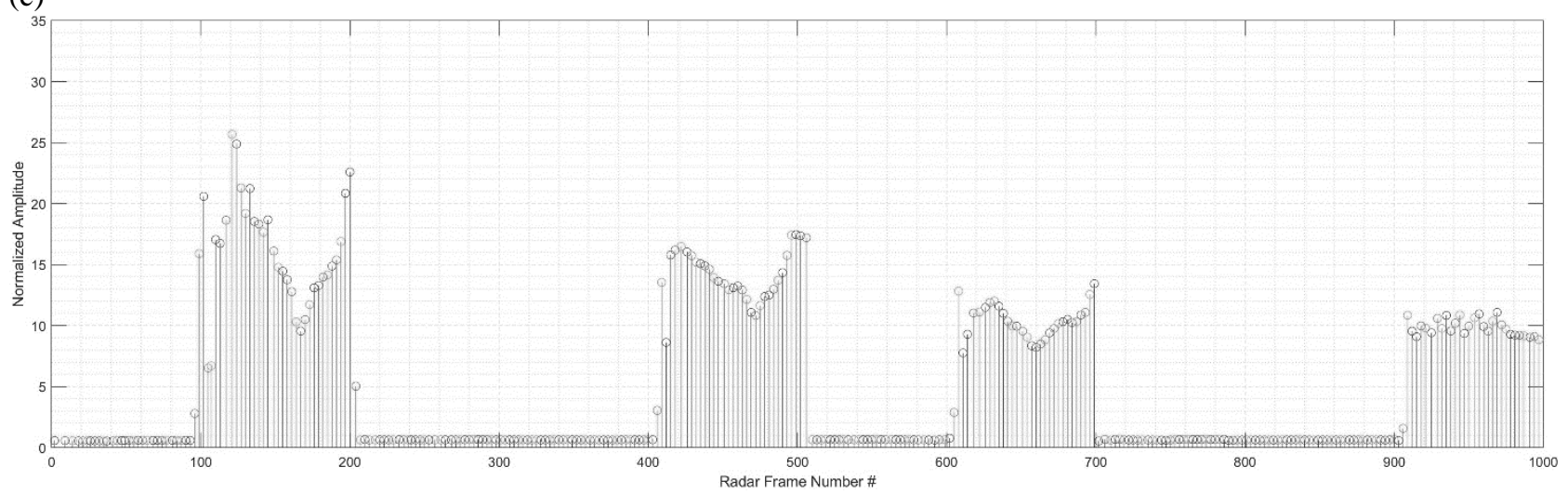

Fig. 11 - (a) 100 radar frames intervals with on off movement, (b) 200 radar frames intervals with on off movement (c) $100 \& 200$ radar frame intervals with on off movement 
(a)

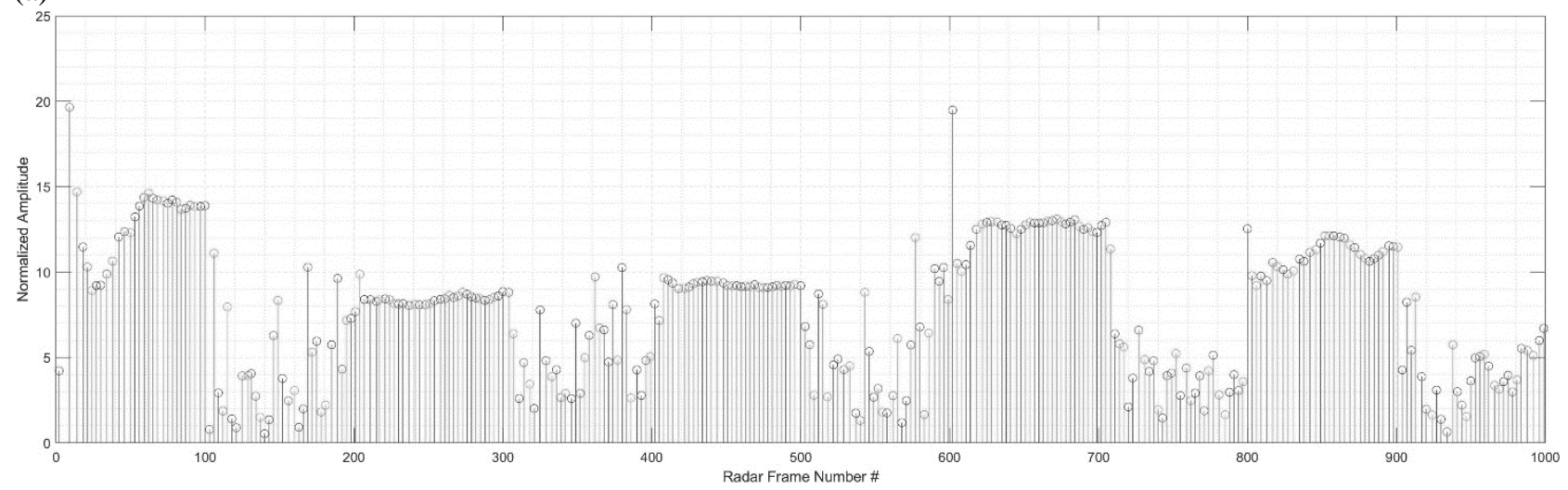

(b)

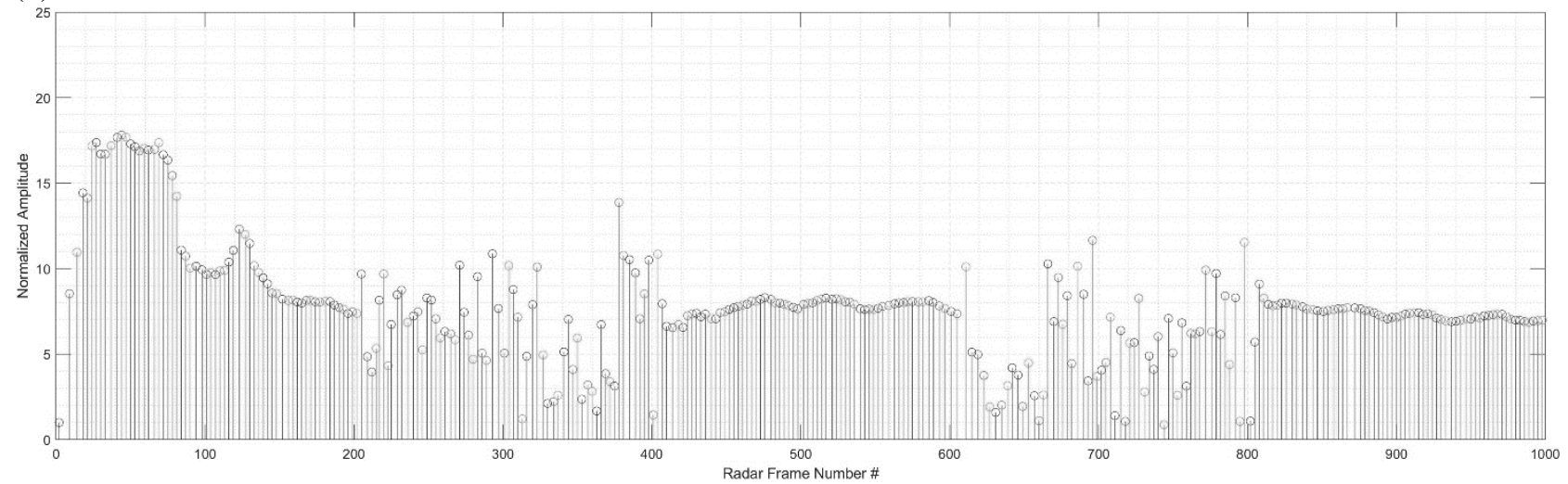

(c)

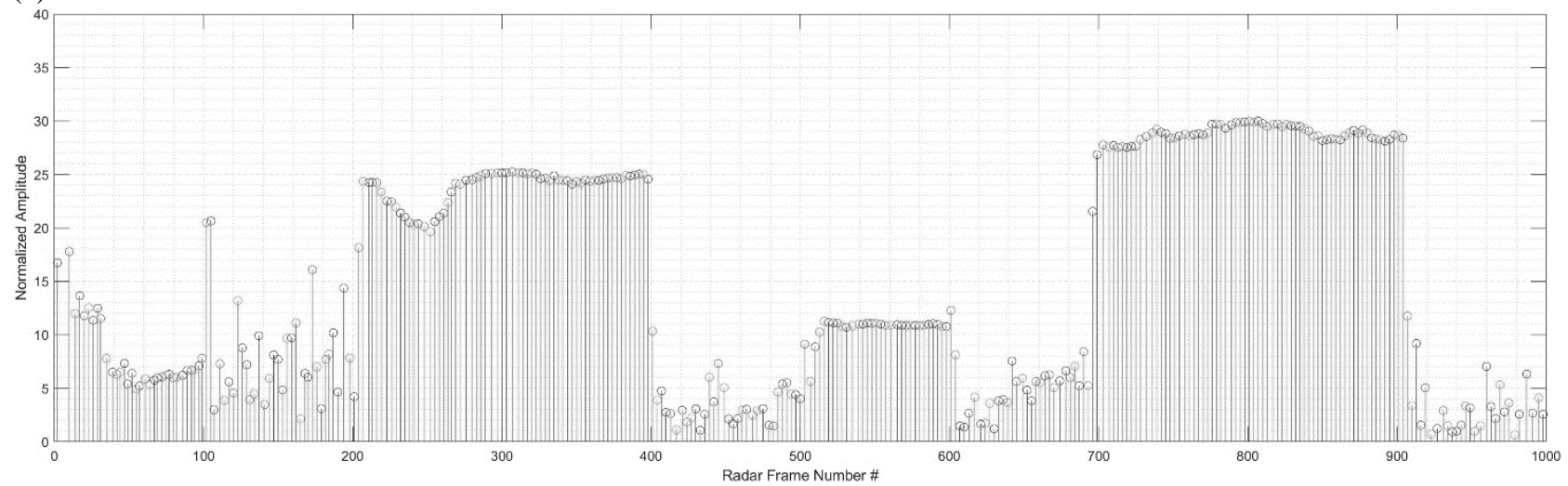

Fig. 12 - (a) 100 radar frames intervals with fingers wiggling movement, (b) 200 radar frames intervals with fingers wiggling movement (c) 100 \& 200 radar frame intervals with fingers wiggling movement

\section{Conclusion}

In this paper, a work on detecting fine human motion using IR-UWB radar sensor is presented. In this preliminary work, the movement of hand and fingers can be discriminated accurately at a distance of between $5 \mathrm{~cm}$ to $10 \mathrm{~cm}$. However, the present of noises at some data points which cause the amplitudes of several radar frames behaves radically has to be addressed in future works. Therefore, further improvement and other consideration to suppress and reduce noises will be implemented on the system model in future works. 


\section{Acknowledgement}

The authors would like to thank Universiti Malaysia Sabah (UMS) for funding this study under the research grants; UMSGreat/GUG00244-1-2018.

\section{References}

[1] Skolnik, M. I. (1962). Introduction to radar. Radar Handbook 2

[2] Watson-Watt, R. (1945). Radar in war and in peace. Nature 155, 3935, 319-324

[3] A. Yarovoy, L. Ligthart, J. Matuzas, and B. Levitas (2006). UWB radar for human being detection, IEEE Aerosp. Electron. Syst. Mag., vol. 21, no. 3, 10-14

[4] B. Lee, S. Lee, Y. Yoon, K. Park and S. Kim. (2017). Adaptive clutter suppression algorithm for human detection using IR-UWB radar, 2017 IEEE SENSORS (pp. 1-3). Glasgow

[5] E. C. Lee and S. H. Cho. (2016). Performance enhancement of multi-human detection using an IR-UWB radar by an adaptive thresholding algorithm. 2016 IEEE International Conference on Network Infrastructure and Digital Content (IC-NIDC) (pp. 476-480). Beijing

[6] S. Yoo, S. Chung, D. Seol and S. H. Cho. (2018). Adaptive Clutter Suppression Algorithm for Detection and Positioning using IR-UWB Radar. 2018 9th International Conference on Ultrawideband and Ultrashort Impulse Signals (UWBUSIS) (pp. 40-43). Odessa

[7] T. Ha and J. Kim. (2019). Detection and Localization of Multiple Human Targets Based on Respiration Measured by IR-UWB Radars. 2019 IEEE SENSORS (pp. 1-4). Montreal, QC, Canada

[8] S. Lee and J. Seo. (2018). IR-UWB radar-based near-field head rotation movement sensing under fixed body motions. 2018 International Conference on Electronics, Information, and Communication (ICEIC) (pp. 1-3). Honolulu, HI

[9] M. Staderini. (2002). UWB radars in medicine. IEEE Aerospace and Electronic Systems Magazine (vol. 17, no. 1, pp. 13-18)

[10] X. Li, S. K. Davis, S. C. Hagness, D. W. Van Der Weide, and B. D.Van Veen. (2004). Microwave imaging via space-time beamforming: Experimental investigation of tumor detection in multilayer breast phantoms. IEEE Trans. Microwave Theory Technology (vol. 52, no. 8, pp. 1856-1865)

[11] M. Converse, E. J. Bond, S. C. Hagness, and B. D. Van Veen. (2004). Ultrawide-band microwave space-time beamforming for hyperthermia treatment of breast cancer: A computational feasibility study. IEEE Trans.Microw. Theory Technology (vol. 52, no. 8, pp. 1876-1889)

[12] V. M. Lubecke, O. Boric-Lubecke, A. Host-Madsen, and A. E. Fathy. (2007). Through-the-wall radar life detection and monitoring. IEEE MTT-S Int. Microw. Symp. Dig. (pp.769-772). Honolulu, HI, USA

[13] M. Davy, T. Lepetit, J. de Rosny, C. Prada, and M. Fink. (2010). Detection and imaging of human beings behind a wall using the DORT method. Progr. Electromagn. Res. (vol. 110, pp. 353-369)

[14] J. Sachs et al. (2008). Detection and tracking of moving or trapped people hidden by obstacles using ultrawideband pseudo-noise radar. Proc. EuRad (pp. 408-411)

[15] A. Lazaro, D. Girbau, and R. Villarino. (2010). Analysis of vital signs monitoring using an IR-UWB radar. Progress in Electromagnetics Research (100: pp. 265-284)

[16] G. Pochanin, S. Masalov, I. Pochanina, L. Capineri, P. Falorni and T. Bechtel. (2016). Modern trends in development and application of the UWB radar systems. 2016 8th International Conference on Ultrawideband and Ultrashort Impulse Signals (UWBUSIS) (pp. 7-11). Odessa

[17] S. Hussain and W. Munir. (2017). UWB BAN antennas: Recent trend and developments. 2017 International Conference on Communication, Computing and Digital Systems (C-CODE) (pp. 86-89). Islamabad

[18] D. Minoli and B. Occhiogrosso. (2018). Ultrawideband (UWB) Technology For Smart Cities IoT Applications. 2018 IEEE International Smart Cities Conference (ISC2) (pp. 1-8). Kansas City, MO, USA 